\title{
Article
}

\section{Effectiveness of Adaptive Silverware on Range of Motion of the Hand}

McDonald, Susan, Levine, David, Richards, Jim and Aguilar, Lauren Available at http://clok.uclan.ac.uk/13484/

McDonald, Susan, Levine, David, Richards, Jim ORCID: 0000-0002-4004-3115 and Aguilar, Lauren (2016) Effectiveness of Adaptive Silverware on Range of Motion of the Hand. PeerJ, 15 (4). e1667-:e166.

It is advisable to refer to the publisher's version if you intend to cite from the work. http://dx.doi.org/10.7717/peerj.1667

For more information about UCLan's research in this area go to http://www.uclan.ac.uk/researchgroups/ and search for < name of research Group>.

For information about Research generally at UCLan please go to http://www.uclan.ac.uk/research/

All outputs in CLoK are protected by Intellectual Property Rights law, including Copyright law. Copyright, IPR and Moral Rights for the works on this site are retained by the individual authors and/or other copyright owners. Terms and conditions for use of this material are defined in the policies page.

\section{CLoK}

Central Lancashire online Knowledge www.clok.uclan.ac.uk

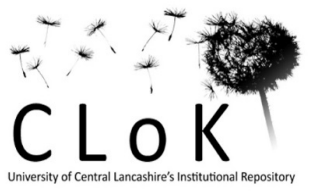




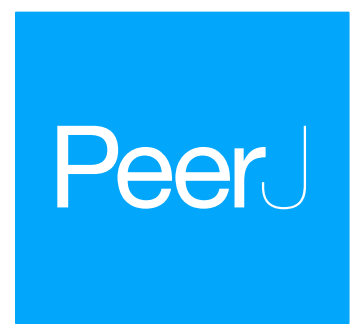

Submitted 1 December 2015 Accepted 17 January 2016 Published 15 February 2016

Corresponding author

David Levine, david-levine@utc.edu

Academic editor

Laura Guidetti

Additional Information and Declarations can be found on page 8

DOI 10.7717/peerj.1667

Copyright

2016 McDonald et al.

Distributed under

Creative Commons CC-BY 4.0

\section{Effectiveness of adaptive silverware on range of motion of the hand}

\author{
Susan S. McDonald ${ }^{1}$, David Levine ${ }^{2}$, Jim Richards ${ }^{3}$ and Lauren Aguilar ${ }^{4}$ \\ ${ }^{1}$ Department of Occupational Therapy, University of Tennessee-Chattanooga, Chattanooga, TN, \\ United States \\ ${ }^{2}$ Department of Physical Therapy, University of Tennessee-Chattanooga, Chattanooga, TN, United States \\ ${ }^{3}$ Allied Health Research unit, University of Central Lancashire, Preston, United Kingdom \\ ${ }^{4}$ Honors College, University of Tennessee-Chattanooga, Chattanooga, TN, United States
}

\section{ABSTRACT}

Background. Hand function is essential to a person's self-efficacy and greatly affects quality of life. Adapted utensils with handles of increased diameters have historically been used to assist individuals with arthritis or other hand disabilities for feeding, and other related activities of daily living. To date, minimal research has examined the biomechanical effects of modified handles, or quantified the differences in ranges of motion (ROM) when using a standard versus a modified handle. The aim of this study was to quantify the ranges of motion (ROM) required for a healthy hand to use different adaptive spoons with electrogoniometry for the purpose of understanding the physiologic advantages that adapted spoons may provide patients with limited ROM.

Methods. Hand measurements included the distal interphalangeal joint (DIP), proximal interphalangeal joint (PIP), and metacarpophalangeal joint (MCP) for each finger and the interphalangeal (IP) and MCP joint for the thumb. Participants were 34 females age 18-30 (mean age $20.38 \pm 1.67$ ) with no previous hand injuries or abnormalities. Participants grasped spoons with standard handles, and spoons with handle diameters of $3.18 \mathrm{~cm}$ (1.25 inch), and $4.45 \mathrm{~cm}$ (1.75 inch). ROM measurements were obtained with an electrogoniometer to record the angle at each joint for each of the spoon handle sizes.

Results. A $3 \times 3 \times 4$ repeated measures ANOVA (Spoon handle size by Joint by Finger) found main effects on ROM of Joint $\left(F(2,33)=318.68\right.$, Partial $\eta^{2}=.95$, $p<.001)$, Spoon handle size $\left(F(2,33)=598.73\right.$, Partial $\left.\eta^{2}=.97, p<.001\right)$, and Finger $\left(F(3,32)=163.83\right.$, Partial $\left.\eta^{2}=.94, p<.001\right)$. As the spoon handle diameter size increased, the range of motion utilized to grasp the spoon handle decreased in all joints and all fingers $(p<0.01)$.

Discussion. This study confirms the hypothesis that less range of motion is required to grip utensils with larger diameter handles, which in turn may reduce challenges for patients with limited ROM of the hand.

Subjects Kinesiology, Orthopedics, Rheumatology

Keywords Hand, Finger, Thumb, Range of motion, Disability, Electrogoniometer, Adaptive equipment, Activities of daily living, Self-care, Arthritis 


\section{INTRODUCTION}

Adaptive equipment is used by approximately $23 \%$ of older adults in the United States, indicating the importance of validating the efficacy and effectiveness of these assistive devices for optimal and appropriate evidence-based prescription (Kraskowsky \& Finlayson, 2001). Hand impairment can inhibit or reduce functional ability to perform many activities of daily living such as dressing, bathing, eating, and other self-care. It has been previously reported that the use of traditional utensils to feed oneself can be difficult and/or painful with impaired hand function (Brach et al., 2002). Objective assessment of hand joint range of motion (ROM) required for functional activities can be valuable in prescribing adaptive equipment for individuals with impairments. A person with normal hand ROM should not feel discomfort in performing tasks such as gripping a standard sized eating utensil; the same task, however, can be difficult if hand range of motion is limited due to either injury or disability. Examples of conditions that commonly affect hand ROM include stroke, osteoarthritis, rheumatoid arthritis, and cerebral palsy (Van Roon \& Steenbergen, 2006). According to the Arthritis Foundation (2015), 1 in 5 adults in the United States are affected by arthritis, indicating a great demand for methods to relieve associated complications. A common intervention consists of using increased diameter grip handles on eating utensils. These grips are typically made from a foam-like material and are available in varying sizes such as $3.18 \mathrm{~cm}(1.25 \mathrm{inch})$ and $4.45 \mathrm{~cm}(1.75$ inch) diameters as seen in Fig. 1.

Although adaptive utensils with modified handles are commonly used, limited research quantifies the biomechanical effects of larger grips or describes how modified handles affect the ROM of hand joints. Of the prescribed eating and drinking adaptive devices, patients were found to not use $35 \%$ of them (Neville-Jan et al., 1993). Primary reasons for this noncompliance likely stem from the improper sizing of recommended device (Kraskowsky \& Finlayson, 2001; Neville-Jan et al., 1993). An in-depth review of the literature by Thomas, Pinkelman \& Gardine (2010) found the four most common reasons for non-compliance for using adaptive equipment are: (1) the patient was not included in deciding on adaptive equipment; (2) inadequate instructions were given; (3) the medical condition improves so they no longer need the adaptive equipment; and (4) the patient's environment is favorable to their condition so they no longer need the adaptive equipment. An individualized approach for prescribing assistive equipment that improves the quality of life for clients mirrors the client-centeredness of rehabilitation therapists. A client-centered approach to assistive equipment provision requires client input when deciding on equipment and to ensure its relevance and appropriateness for the client (Hoffmann \& McKenna, 2004). Determining the individuals ROM can help with adaptive equipment prescription and may decrease pain associated with simple tasks of daily life and improve utilization and evidence-based rehabilitation outcomes. Bazanski (2010) suggested that a $50^{\circ}$ lack of flexion in metacarpophalangeal joints, the most important joints during grip, causes a $24 \%$ increase in finger impairment.

Electrogoniometers have previously been found to be a valid and reliable tool for the measurement of ROM (Bronner, Agraharasamakulam \& Ojofeitimi, 2010; Carnaz et al., 


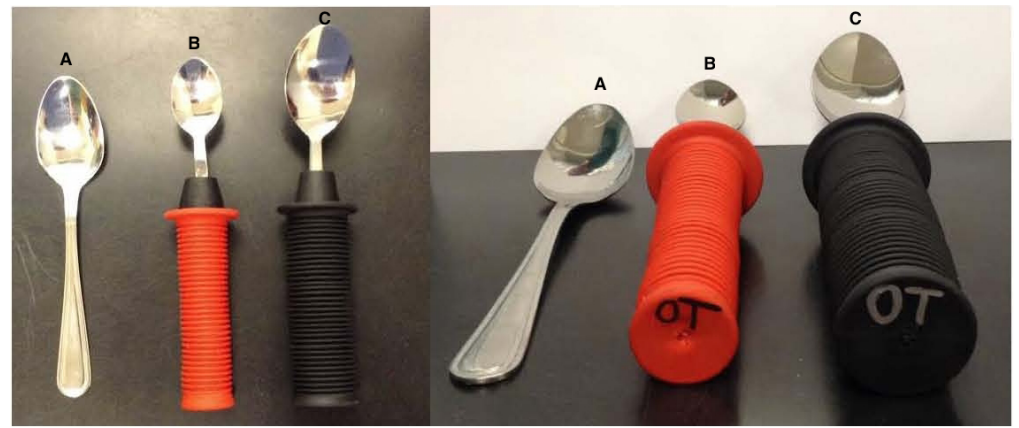

Figure 1 Adaptive utensils with modified handles. These images depict a standard spoon (A), a spoon with a $3.18 \mathrm{~cm}(1.25 \mathrm{inch})$ diameter handle (B), and a spoon with a $4.45 \mathrm{~cm}$ diameter handle $(1.75 \mathrm{inch})$ (C).

2013; Piriyaprasarth et al., 2008). One previous study used a biaxial goniometer to analyze thumb movements during the use of hand held devices, such as mobile phones, and found the electrogoniometer to be both clinically feasible and accurate (Jonsson, Johnson \& Hagberg, 2007).

Modified spoon handles can be beneficial while feeding and research has shown positive outcomes regarding the potential benefits of these utensils for patients with conditions including rheumatoid arthritis, Parkinson's disease, and cerebral palsy (Ma et al., 2008; Van Roon \& Steenbergen, 2006). Handle diameter and its relationship to spoon-use movement was examined in patients with Parkinson's disease. Handles of small $(1.2 \mathrm{~cm})$, medium $(2.0 \mathrm{~cm})$, and large $(3.8 \mathrm{~cm})$ diameter size were studied and the large handles significantly decreased task movement time and subjective scores of comfort and feasibility of use ( $\mathrm{Ma}$ et al., 2008). This was likely seen as the hand aperture of the participants with Parkinson's disease was significantly smaller than that of the controls. This study provides evidence of the benefits of altering handle size, but accounts for only the overall movement of the hand as a single unit, and does not address how the grip affects individual joints within the hand.

The use of modified handles for daily activities in persons with rheumatoid arthritis suggests that these assistive devices can help to protect joint integrity by minimizing joint forces and avoiding tight grips (Shipham \& Pitout, 2003). Van Roon \& Steenbergen (2006) examined spoon grip-size and its effects on movement kinematics and food spilling for patients with cerebral palsy. Participants with tetraparesis performed quicker transportation of water from one bowl to another and with less spillage when using a $5 \mathrm{~cm}$ ( 2 inch) diameter modified spoon versus a $3 \mathrm{~cm}$ ( 1.18 inch) and $1 \mathrm{~cm}(0.40$ inch $)$ spoon.

While these studies show benefits that may result from using modified spoon handles, they do not study biomechanical changes that occur to individual finger joints when gripping the handles. This study aimed to determine the biomechanical differences in ROM of the fingers when using three different spoon handles in young healthy subjects. These included a standard spoon, a $3.18 \mathrm{~cm}$ (1.25 inch) diameter modified handle and a $4.45 \mathrm{~cm}$ (1.75 inch) diameter modified handle. These sizes were chosen as they are commonly adopted by patients among those commercially available. The purpose of this 
study was to determine differences in the ROM required from the joints in the hand when gripping three different sizes of adaptive spoon handles with various diameters.

\section{MATERIALS \& METHODS}

\section{Subjects}

Thirty-four healthy females who were students at the University of Tennessee at Chattanooga, between the ages of 18 and $30(x=20.38 \pm 1.67)$ years of age, voluntarily participated in this study. The average grip strength was $58.41 \mathrm{psi}$, consistent with previously published normative values for females between the ages of 20-29 (Bohannon, 2006; Peters et al., 2011).

Exclusion criteria included previous hand injury, any neurological condition that would impair hand movement, arthritis or any other condition that would prevent the subject from having normal hand function and ROM. To reduce the amount of variables potentially affecting or influencing results, all participants were right handed and only the dominant sides were assessed, as the dominant hand is typically used to grasp utensils. All subjects read and signed an informed consent form in accordance with the Institutional Review Board at the University of Tennessee at Chattanooga (IRB \#14-026). There were no incentives or rewards given for participating. Subjects were recruited using online advertisements sent to students of the University of Tennessee at Chattanooga.

\section{Equipment}

A Jamar hydraulic hand dynamometer (Patterson Medical, Warrenville, IL, USA) was used to take a total of 3 measurements of grip strength, which were averaged. The electrogoniometer utilized (Biometrics Ltd, Ladysmith, VA, USA) was comprised of an angle display unit and a single axis goniometer with accuracy previously reported as $\pm 0.1^{\circ}$ (Christensen, 1999). A foam arm rest (Fig. 2) was used to provide a comfortable standardized position for the subjects during data collection.

\section{Experimental protocol}

Subjects were seated with their shoulder in the anatomical position, and their elbow at a $90^{\circ}$ angle, with the hand dynamometer handle placed in the second grip position which is recognized as the standard position for producing the most accurate results (Massy-Westropp et al., 2011; Trampisch et al., 2012). Grip strength was tested by asking participants to maintain a maximal isometric contraction for $3 \mathrm{~s}$. Participants then placed their right arm on a foam armrest to standardize arm position (Fig. 2). A single axis electrogoniometer was used to measure the angles created at each joint of the hand (Fig. 3).

For all finger joint measurements subjects were given the three spoons (standard handles, and handle diameter of $3.18 \mathrm{~cm}$ ( $1.25 \mathrm{inch})$, and handle diameter of $4.45 \mathrm{~cm}(1.75 \mathrm{inch}))$ in randomized order and instructed to grip the spoon as if they were going to feed themselves while keeping all fingers in contact with the spoon. In order to confirm that the subjects maintained a solid grip on the spoon throughout the experiment, a small lightweight object was placed in the spoon to ensure they could lift and balance an object with their grip. Hand measurements included the distal interphalangeal joint (DIP), proximal interphalangeal 


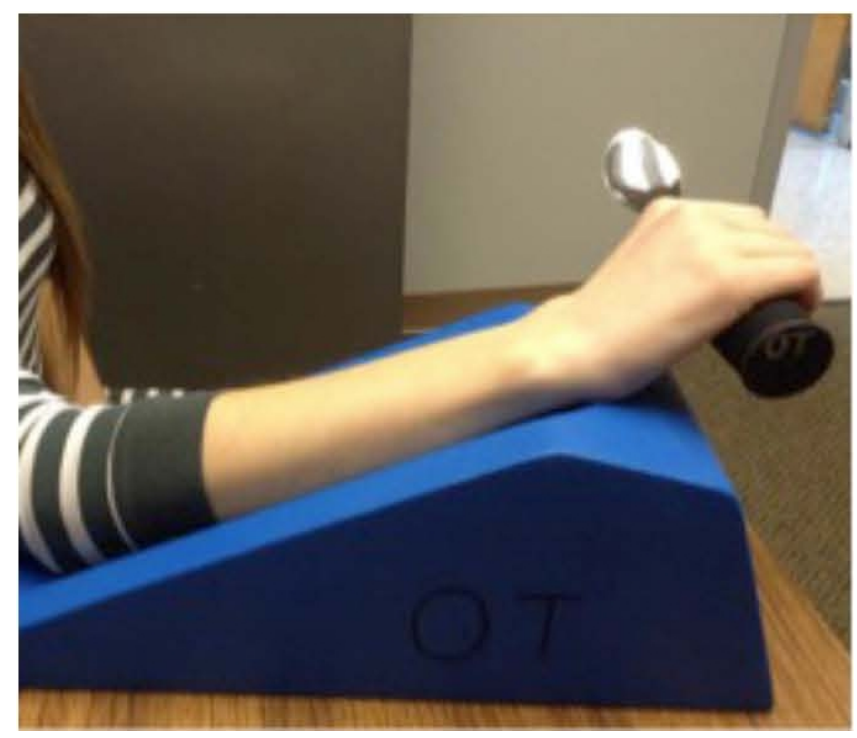

Figure 2 Foam arm rest to support the forearm.

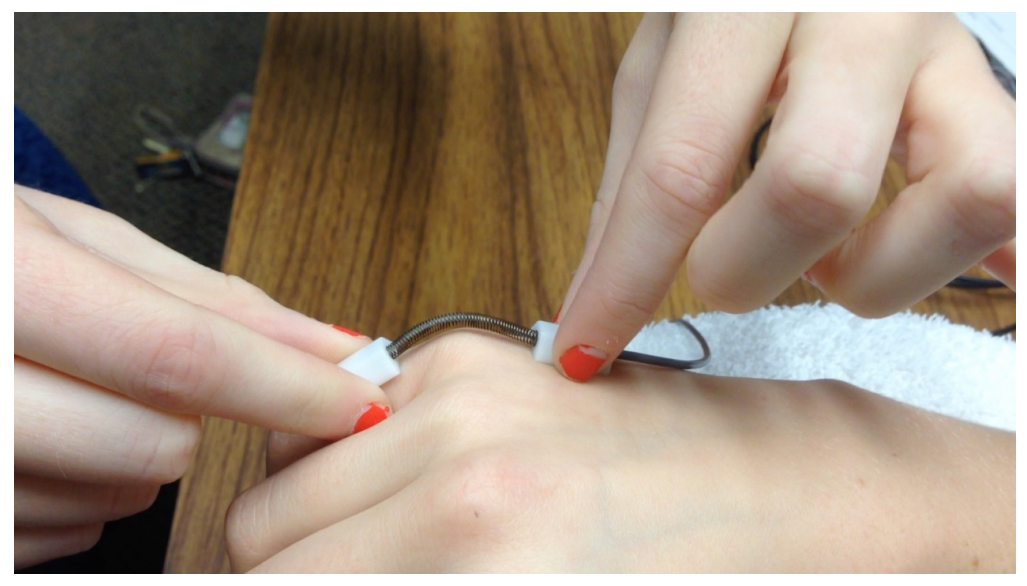

Figure 3 Single axis electrogoniometer measuring flexion of the fifth (pinky) finger metacarpophalangeal (MCP) joint. Image is demonstrating measuring the MCP joint of the pinky finger. Sensor ' $A$ ' is placed on the metacarpal shaft and sensor ' $\mathrm{B}$ ' is placed on the proximal phalanx. (Source: Goniometer and Torsiometer Operating Manual. Biometrics Ltd.)

joint (PIP), and metacarpophalangeal joint (MCP) for each finger and the interphalangeal (IP) and MCP joint for the thumb. Measurements were obtained for all joints and all fingers by placing one sensor on the proximal bone and one sensor on the distal bone adjacent to the joint being measured (Fig. 3 displays an example of the pinky finger MCP). The angle was displayed on the display unit and was recorded. All measurements were made in triplicate. 


\begin{tabular}{|c|c|c|}
\hline & MCP & IP \\
\hline Standard handle & $30.62^{\circ} \pm 16.08^{\circ}$ & $45.7^{\circ} \pm 19.61^{\circ}$ \\
\hline $3.18 \mathrm{~cm}$ (1.25 inch) handle & $26.46^{\circ} \pm 14.50^{\circ \mathrm{a}}$ & $42.28^{\circ} \pm 10.93^{\circ \mathrm{a}}$ \\
\hline $4.45 \mathrm{~cm}$ (1.75 inch) handle & $16.53^{\circ} \pm 14.57^{\circ \mathrm{a}, \mathrm{b}}$ & $36.43^{\circ} \pm 12.13^{\circ \mathrm{a}, \mathrm{b}}$ \\
\hline
\end{tabular}

Notes.

${ }^{\mathrm{a}}$ Difference between modified handles and standard handle $(P<0.01)$.

${ }^{\mathrm{b}}$ Difference between 3.18 and $4.45 \mathrm{~cm}$ handles $(P<0.01)$.

Table 2 Comparison of index finger (second digit) ROM using a standard spoon, and two commercial spoons with enlarged diameter handles $(3.18 \mathrm{~cm}(1.25$-inch $)$ and $4.45 \mathrm{~cm}(1.75$-inch $))$.

\begin{tabular}{llll} 
& MCP & PIP & DIP \\
\hline Standard handle & $87.47^{\circ} \pm 12.12^{\circ}$ & $106.59^{\circ} \pm 7.70^{\circ}$ & $63.58^{\circ} \pm 11.33^{\circ}$ \\
$3.18 \mathrm{~cm}(1.25$ inch) handle & $56.98^{\circ} \pm 13.28^{\circ \mathrm{a}}$ & $70.73^{\circ} \pm 6.36^{\circ \mathrm{a}}$ & $45.86^{\circ} \pm 6.80^{\circ \mathrm{a}}$ \\
$4.45 \mathrm{~cm}(1.75$ inch) handle & $40.68^{\circ} \pm 11.77^{\circ \mathrm{a}, \mathrm{b}}$ & $55.01^{\circ} \pm 8.13^{\text {oa, b }}$ & $35.59^{\circ} \pm 6.96^{\text {oa, b }}$ \\
\hline
\end{tabular}

Notes.

${ }^{\mathrm{a}}$ Difference between modified handles and standard handle $(P<0.01)$.

${ }^{\mathrm{b}}$ Difference between 3.18 and $4.45 \mathrm{~cm}$ handles $(P<0.01)$.

Table 3 Comparison of middle finger (third digit) ROM using a standard spoon, and two commercial spoons with enlarged diameter handles $(3.18 \mathrm{~cm}(1.25$-inch $)$ and $4.45 \mathrm{~cm}(1.75$-inch $))$.

\begin{tabular}{llll} 
& MCP & PIP & DIP \\
\hline Standard handle & $93.66^{\circ} \pm 10.12^{\circ}$ & $104.53^{\circ} \pm 5.51^{\circ}$ & $71.31^{\circ} \pm 11.01^{\circ}$ \\
$3.18 \mathrm{~cm}(1.25$ inch $)$ handle & $67.42^{\circ} \pm 12.89^{\circ \mathrm{a}}$ & $67.1^{\circ} \pm 5.78^{\circ \mathrm{a}}$ & $50.93^{\circ} \pm 7.07^{\circ \mathrm{a}}$ \\
$4.45 \mathrm{~cm}(1.75 \mathrm{inch})$ handle & $52.98^{\circ} \pm 12.23^{\circ \mathrm{a}, \mathrm{b}}$ & $53.68^{\circ} \pm 4.94^{\circ \mathrm{aa}, \mathrm{b}}$ & $39.71^{\circ} \pm 7.43^{\mathrm{oa}, \mathrm{b}}$ \\
\hline
\end{tabular}

Notes.

${ }^{\mathrm{a} D}$ Difference between modified handles and standard handle $(P<0.01)$.

${ }^{\mathrm{b}}$ Difference between 3.18 and $4.45 \mathrm{~cm}$ handles $(P<0.01)$.

\section{RESULTS}

Mean values and standard deviations of ROM are reported for each finger, by joint and spoon handle size (Tables 1-5). A $3 \times 3 \times 4$ repeated measures ANOVA (Spoon handle size by Joint by Finger) found main effects on ROM of Joint $\left(F(2,33)=318.68\right.$, Partial $\eta^{2}=.95$, $p<.001)$, Spoon handle size $\left(F(2,33)=598.73\right.$, Partial $\left.\eta^{2}=.97, p<.001\right)$, and Finger $\left(F(3,32)=163.83\right.$, Partial $\left.\eta^{2}=.94, p<.001\right)$. Pairwise comparisons showed that as spoon size increased, the range of motion needed decreased in all joints and all fingers $(p<0.01)$. In all five fingers the differences in ROM between the standard spoon and both adaptive spoons was statistically significant $(p<0.01$ ), with the adaptive spoons requiring less ROM for grasp. In all five fingers the difference in ROM between the $3.18 \mathrm{~cm}$ (1.25 inch) diameter and $4.45 \mathrm{~cm}$ ( $1.75 \mathrm{inch})$ diameter spoons was statistically significant $(p<0.01)$ with the $4.45 \mathrm{~cm}$ (1.75 inch) diameter spoon requiring less ROM for grasp (Tables 1-5). 


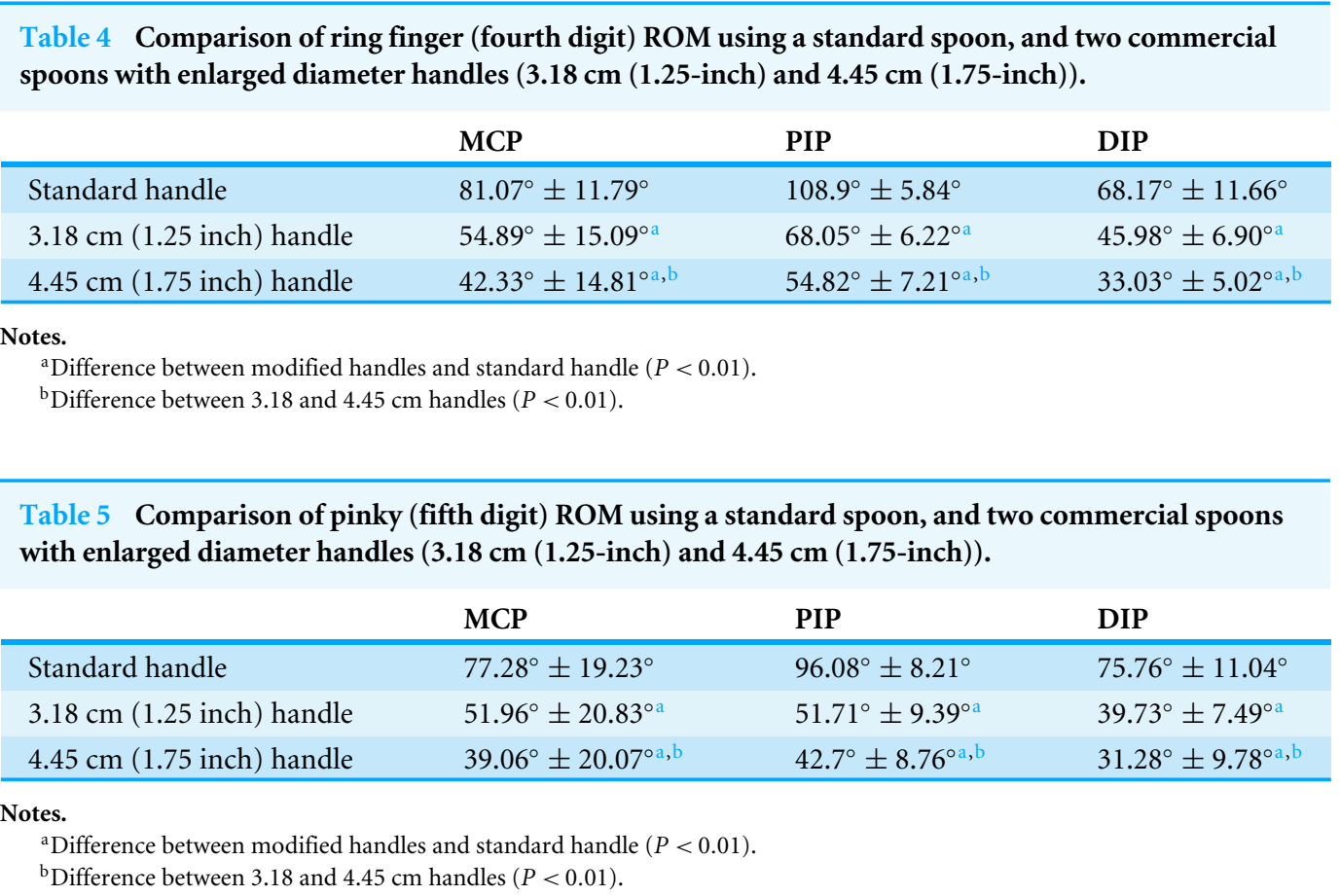

\section{DISCUSSION}

This study quantified finger and thumb joint ROM needed for healthy adult females to grip a standard spoon and two different adaptive spoon handle sizes. A statistical comparison between the ROM for each finger, for each of the three spoons showed a significant difference between the angles formed at each joint, with respect to the spoon handle size. The angle recorded can be thought of as the distance the joint moved from its original position in order to grasp the spoon handle. Joint angles were greater when subjects gripped the standard spoon handle compared to the handles of the modified spoons. The need for greater ROM with a standard spoon indicates a potential challenge for someone with limited hand ROM to grasp a standard sized spoon handle.

The variability of the data obtained for hand ROM was actually smaller than expected (Tables 1-5). Some variability between individuals was likely due to the variations in which people grasp a utensil despite standardized instructions being given or holding the spoon. The data listed in Tables 1-5 and the statistical analyses confirm less range of motion is required to grip spoons with modified handles. Patients who benefit from the use of such utensils include those diagnosed with conditions that commonly restrict hand ROM, such as patients diagnosed with carpal tunnel, stroke, cerebral palsy, or rheumatoid arthritis (Van Roon \& Steenbergen, 2006) as well as older adults (Kraskowsky \& Finlayson, 2001). Knowing the ROM required by the hand to attain certain grasps may help reduce trial-and-error approach and improve the prescription of ADL utensils and could be a clinically relevant consideration for occupational therapists who often fit patients with such assistive devices. 


\section{Future research}

The aim of this study was to provide quantifiable data to support the common practice of employing adaptive equipment such as spoons with increased handle diameter to reduce ROM required to grip a standard spoon handle and thereby increase independence with feeding activities of daily living. Although this concept was successfully confirmed, different research hypotheses could be formed and tested using similar methods. For example, information recorded during the data collection process such as measurements of hand size could be investigated to show possible correlations between variables of hand size and the range of motion required to grip the different spoon handle diameters. This would require interpretation of individual results as opposed to the overall group analysis run for this particular study. Advances in biomodeling may present the opportunity to provide custom silverware and other tools based on the individual's hand size, strength, and functional needs. Other variables could be introduced such as questioning the subject for a subjective rating of comfort to establish what may be the ideal handle size as decreased ROM does not necessarily correlate to increased comfort levels or increased efficiency. A more diverse study population including patients with hand deficits likely to use adaptive equipment could be included in future studies. Certain variables such as grip strength may also be a factor in determining the effectiveness of adaptive utensils when the study population has pre-existing hand impairment, as grip strength performance is highly related to the ability of a subject to use their hand functionality

\section{CONCLUSIONS}

The study quantified the hand range of motion needed for adults to use a standard spoon and two commonly available commercial adaptive spoons. It was hypothesized that it would require less range of motion to grip the spoons with modified handles. An electrogoniometer was used to determine range of motion data for 34 healthy subjects. Statistical analysis found significant differences in range of motion requirements between spoon handle sizes and confirmed the hypothesis that less range of motion is required to grip the modified utensils.

\section{ADDITIONAL INFORMATION AND DECLARATIONS}

\section{Funding}

This study was supported by a Provost student research award from The University of Tennessee at Chattanooga. The funders had no role in study design, data collection and analysis, decision to publish, or preparation of the manuscript.

\section{Grant Disclosures}

The following grant information was disclosed by the authors:

Provost student research award.

\section{Competing Interests}

David Levine is an Academic Editor for PeerJ. 


\section{Author Contributions}

- Susan S. McDonald conceived and designed the experiments, performed the experiments, contributed reagents/materials/analysis tools, wrote the paper, prepared figures and/or tables, reviewed drafts of the paper.

- David Levine and Lauren Aguilar conceived and designed the experiments, performed the experiments, analyzed the data, contributed reagents/materials/analysis tools, wrote the paper, prepared figures and/or tables, reviewed drafts of the paper.

- Jim Richards analyzed the data, contributed reagents/materials/analysis tools, wrote the paper, prepared figures and/or tables, reviewed drafts of the paper.

\section{Human Ethics}

The following information was supplied relating to ethical approvals (i.e., approving body and any reference numbers):

Institutional Review Board at the University of Tennessee at Chattanooga (IRB \#14-026).

\section{Data Availability}

The following information was supplied regarding data availability:

Raw data can be found in the Supplemental Information.

\section{Supplemental Information}

Supplemental information for this article can be found online at http://dx.doi.org/10.7717/ peerj.1667\#supplemental-information.

\section{REFERENCES}

Arthritis Foundation. 2015. Arthritis facts. Available at http://www.arthritis.org/aboutarthritis/understanding-arthritis/what-is-arthritis.php, http://hprints.org/hprints00714715 (accessed 25 November 2015).

Bazanski T. 2010. Metacarpophalangeal joint kinematics during a grip of everyday objects using the three-dimensional motion analysis system. Acta of Bioengineering and Biomechanics 12:79-85.

Bohannon RW. 2006. Hand-held dynamometry: adoption 1900-2005. Perceptual and Motor Skills 103(1):3-4.

Brach JS, VanSwearingen JM, Newman AB, Kriska AM. 2002. Identifying early decline of physical function in community-dwelling older women: performance-based and self-report measures. Physical Therapy 82:320-328.

Bronner S, Agraharasamakulam S, Ojofeitimi S. 2010. Reliability and validity of electrogoniometry measurement of lower extremity movement. Journal of Medical Engineering and Technology 34:232-242 DOI 10.3109/03091900903580512.

Carnaz L, Moriguchi CS, De Oliveira AB, Santiago PR, Caurin GA, Hansson GA, Coury HJ. 2013. A comparison between flexible electrogoniometers, inclinometers and three-dimensional video analysis system for recording neck movement. Medical Engineering \& Physics 35:1629-1637 DOI 10.1016/j.medengphy.2013.05.014. 
Christensen HW. 1999. Precision and accuracy of an electrogoniometer. Journal of Manipulative and Physiological Therapeutics 22:10-14 DOI 10.1016/S0161-4754(99)70099-0.

Hoffmann T, McKenna K. 2004. A survey of assistive equipment use by older people following hospital discharge. The British Journal of Occupational Therapy 67:75-82 DOI 10.1177/030802260406700204.

Jonsson P, Johnson PW, Hagberg M. 2007. Accuracy and feasibility of using an electrogoniometer for measuring simple thumb movements. Ergonomics 50:647-659 DOI 10.1080/00140130601164490.

Kraskowsky LH, Finlayson M. 2001. Factors affecting older adults' use of adaptive equipment: review of the literature. American Journal of Occupational Therapy 55:303-310 DOI 10.5014/ajot.55.3.303.

Ma HI, Hwang WJ, Chen-Sea MJ, Sheu CF. 2008. Handle size as a task constraint in spoon-use movement in patients with Parkinson's disease. Clinical Rehabilitation 22:520-528 DOI 10.1177/0269215507086181.

Massy-Westropp NM, Gill TK, Taylor AW, Bohannon RW, Hill CL. 2011. Hand grip strength: age and gender stratified normative data in a population-based study. $B M C$ Research Notes 4:127 DOI 10.1186/1756-0500-4-127.

Neville-Jan A, Piersol C, Kielhofner G, Davis K. 1993. Adaptive equipment: a study of utilization after hospital discharge. Occupational Therapy in Health Care 8(4):3-18 DOI 10.1300/J003v08n04_02.

Peters MJ, Van Nes SI, Vanhoutte EK, Bakkers M, Van Doorn PA, Merkies IS, Faber CG. 2011. Revised normative values for grip strength with the Jamar dynamometer. Journal of the Peripheral Nervous System 16:47-50

DOI 10.1111/j.1529-8027.2011.00318.x.

Piriyaprasarth P, Morris ME, Winter A, Bialocerkowski AE. 2008. The reliability of knee joint position testing using electrogoniometry. BMC Musculoskeletal Disorders 9:6 DOI 10.1186/1471-2474-9-6.

Shipham I, Pitout SJ. 2003. Rheumatoid arthritis: hand function, activities of daily living, grip strength and essential assistive devices. Curationis 26:98-106.

Thomas WN, Pinkelman LA, Gardine CJ. 2010. The reasons for noncompliance with adaptive equipment in patients returning home after a total hip replacement. Physical and Occupational Therapy in Geriatrics 28:170-180 DOI 10.3109/02703181003698593.

Trampisch US, Franke J, Jedamzik N, Hinrichs T, Platen P. 2012. Optimal Jamar dynamometer handle position to assess maximal isometric hand grip strength in epidemiological studies. Journal of Hand Surgery. American Volume 37:2368-2373 DOI 10.1016/j.jhsa.2012.08.014.

Van Roon D, Steenbergen B. 2006. The use of ergonomic spoons by people with cerebral palsy: effects on food spilling and movement kinematics. Developmental Medicine and Child Neurology 48:888-891 DOI 10.1017/S0012162206001940. 